\title{
The Design of Electrical Controlling Core for The Chinese Medicine Steam Heat Treatment Device
}

\author{
Jiang Mu-yuan Wang Yu-duo \\ Beijin information science \& Technology University. Beijin, 100101
}

\begin{abstract}
Keywords: temperature control, centralized output; traditional Chinese medicine fumigation; heat treatment device; analgesic and anti-inflammatory.
\end{abstract}

\begin{abstract}
As our material life level unceasing enhancement, people's health consciousness and Care awareness has greatly improved compared with the original. At the same time with the aging of the population of China, the health situation and health awareness of the elderly is also deeply concerned by the society. But we traditional treating lumbocrural pain and cold diarrhea disease clinical therapy method in physiotherapy will occur Liquid steam output terminal area open, resulting in uneven distribution of the liquid, the temperature is not constant, limited efficacy, resulting in low utilization of the drug, at the same time, the medicine steam output terminal can only carry on the parallel movement, the treatment scope is limited, and the instrument is not easy to carry .Therefore, it is imperative to develop a new clinical therapeutic instrument. We will be based on the traditional Chinese medicine fumigation treatment, and develop a more humane intelligent physical therapy fumigation system. In this paper, we have developed a system of heat treatment for hospitals and communities. This system mainly consists of two systems: the first is the temperature control system; and the second is the traditional Chinese medicine steam transmission system, Among them, the temperature control system is the key parts in the study of this paper. In this paper, the overall design of the traditional Chinese medicine fumigation apparatus and specific design of each part are all introduced in detail.
\end{abstract}

\section{Introduction}

\section{Research Background}

With the continuous development of society, as well as the rhythm of our life, the living standard has been greatly improved, people's health consciousness is more and more strong. The current health service has gradually transformed from the influence of epidemic and infectious disease prevention into solving some of the diseases caused by overwork in modern society, and a series of old age diseases caused by the loss of calcium .Population aging is a global trend of development and the old people's osteoporosis, tissue degeneration, bone hyperplasia, chronic diseases become more and more general. So the development of a new type of medical care and disease treatment of physical therapy device system, to achieve the expansion of the function of the hospital, in the true sense of the real increase in the quality of life of the people ,and to help solve the problem of difficult medical treatment.

\section{The current research status of physical therapy device}

Clinical physical therapy system is an important branch of the treatment of diseases such as the waist and back pain, with its unique advantages, the world's governments and research institutions are highly concerned. Current treatment methods are: Traditional Chinese medicine type electric heating treatment, Chinese medicine heat treatment method, traditional Chinese medicine fumigation treatment, traditional Chinese medicine, magnetic heat treatment method and so on. Electrothermal therapy and magnetic heat therapy in the treatment of treatment need to close to the skin of a patient through electrotherapy, this will be on the human body have a certain degree of risk; In the treatment of electromagnetic wave, there is a certain distance to the surface of the skin, and the distribution of the heat field is not uniform and the depth of treatment is shallow. Using the 
traditional fumigation treatment method, effects is dispersing, drug utilization rate is low, and the temperature control is sensitive. Therefore, it is necessary to develop a clinical physical therapy system which is suitable for our country's situation, and can be treated with the specific conditions of different patients

\section{The Design of Chinese Medicine Steam Heat Treatment Device}

\section{The overall design of the traditional Chinese medicine fumigation instrument}

To study the characteristics and future trends of the current clinical physiotherapy instrument, We present a new type of physiotherapy apparatus, which can be treated in a community or hospital. The device is composed of two parts, the steam temperature control system and the traditional Chinese medicine steam output control system. The steam temperature control system of traditional Chinese medicine mainly carries on the automatic control of the upper and lower limits of the steam temperature of the terminal, the output system is the delivery of liquid vapor to the patient's pain.

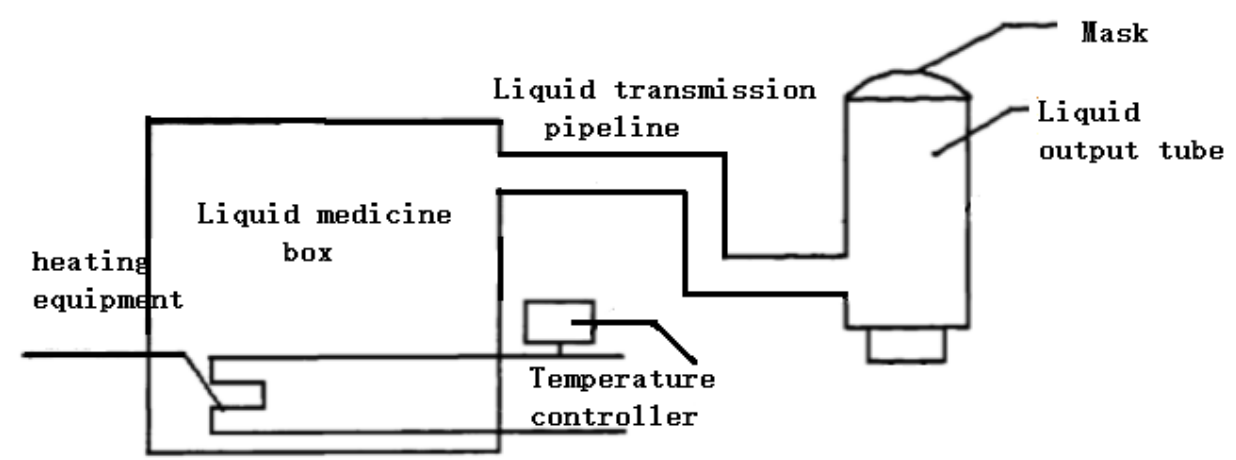

Fig.1 Traditional Chinese medicine fumigation device design

\section{Control system of traditional Chinese medicine fumigation}

As shown in Figure 2, CPU is the use of single-chip control operation, through the CPU, the control circuit module is issued on the request for a break, when the control circuit is connected, heating device module heat the liquid to boil and produce steam, liquid vapor through the steam output port, and temperature measurement circuit for output steam temperature at the same time. If the temperature is too high, the temperature measurement circuit sends out a request to control the single chip microcomputer to send out the instruction, the control circuit is broken; if the temperature is too low, the control circuit will increase the voltage at the ends of the heating device, and make it quick heating. Trigger pulse circuit is used to generate the trigger signal, so that the microcontroller on the control circuit output instruction.

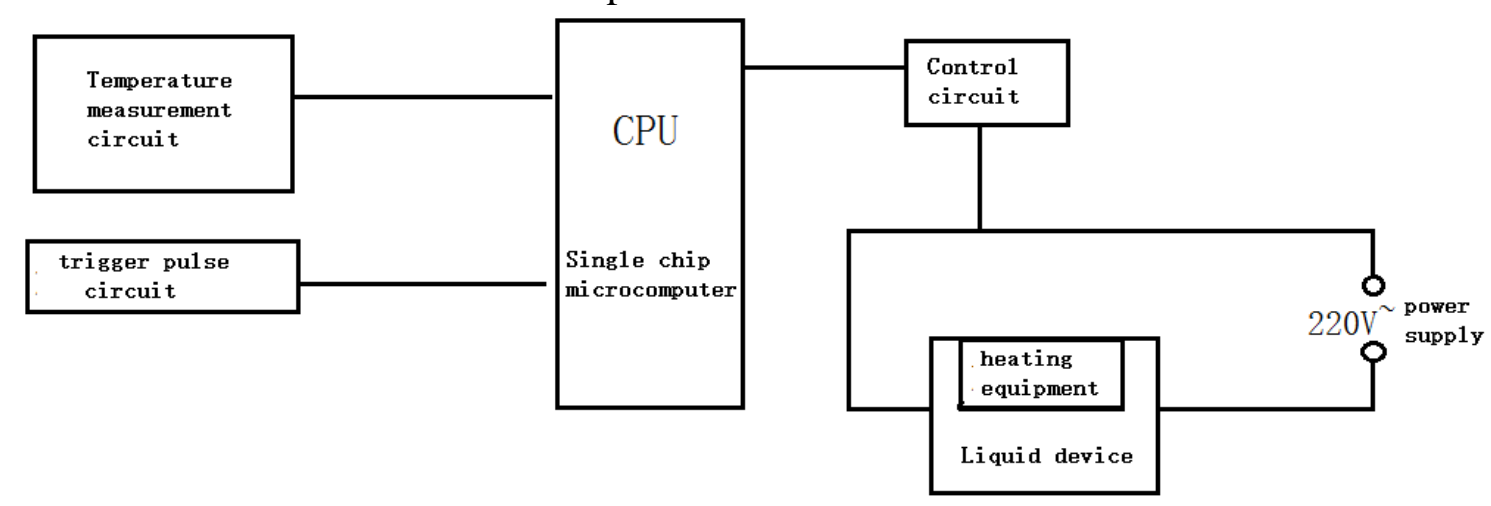

Fig.2 Traditional Chinese medicine fumigation device control system

\section{Hardware design of temperature measurement circuit}

(1) Thermocouple temperature sensor

The use of nickel chromium - nickel silicon thermocouple, the measured temperature range of 0 to 655 DEG c, Cold end compensation using the compensation bridge method, the use of the electric potential to compensate for the unbalanced bridge caused by the cold end temperature 
changes and the thermoelectric potential changes. The unbalanced bridge is composed of resistance $R_{1} 、 R_{2} 、 R_{3} 、 R_{c u}$ 、 four bridge arm and Bridge Road, which is series in the thermocouple circuit, $\mathrm{R}_{c u}$ and the cold end of the thermocouple are both in the same 0 DEG c, $\mathrm{R}_{1}=\mathrm{R}_{2}=\mathrm{R}_{3}=1 \Omega$, Bridge power supply voltage is $4 \mathrm{~V}$, which is powered by a regulated power supply. $R_{s}$ is the current limiting resistor, the resistance is different because of different thermocouple. The bridge is usually balanced at $20^{\circ} \mathrm{C}$, and the resistance of the four leg of the bridge is satisfied with $\mathrm{R}_{1}=$ $\mathrm{R}_{2}=\mathrm{R}_{3}=\mathrm{R}_{c u}$ and the $\mathrm{A}$ and the $\mathrm{B}$ has no output. When the cold end temperature deviates from $20{ }^{\circ} \mathrm{C}$, for example, the $\mathrm{R}_{c u}$ increases when the temperature increases, while the thermoelectric potential decreases with the increase of cold end temperature, $\mathrm{U}_{a b}$ and thermo emf equal quantity reduction, but $\mathrm{U}_{a b}$ and thermoelectric potential superposition of output voltage remains unchanged, so as to achieve the automatic completion of the cold end compensation.

\section{(2) Measurement amplifier circuit}

Actual circuit, the signals from the thermocouple output up to but tens of millivolts $(<30 \mathrm{mV})$, and which contains common mode interference, to solve the circuit amplifying circuit has a high common mode rejection ratio and high gain, low noise and high input impedance, therefore should use measuring amplifier circuit. By the three operational amplifier composed of measuring amplifier, differential input noninverting terminal end $R_{1}$ and $R_{2}$ respectively to $A_{1}$ and $A_{2}$. Because the input impedance is high, we use the symmetrical circuit structure, so that the signal will be directly added to the input, thus ensuring a strong ability to suppress common mode signal. $A_{3}$ is actually a differential follower, the gain is approximately 1 . The amplification factor of the amplifier is: $A_{V}=\frac{V_{0}}{V_{2}-V_{1}}=\frac{R_{F}}{R\left(1+\frac{R_{F 1}+R_{F 2}}{R_{W}}\right)}$ In this circuit, operational amplifier $A_{1}$ and $A_{2}$ performance as long as meet the symmetry conditions, the drift will be greatly reduced, so it is easy to cooperate with the micro output sensor. $\mathrm{R}_{W}$ is used to adjust the amplification factor of the external resistor.

\section{(3) A/D (analog to digital) conversion circuit}

After measuring the amplifier voltage signal, the voltage range of $0 \sim 5 \mathrm{~V}$, the signal is analog signal, the computer can not receive, so it is necessary to carry out A/D conversion . In the actual circuit, using the double integral 12 bit A/D converter ICL7109. The main characteristics are as follows: (1) high accuracy; (2) low noise (3) low drift (4) high input impedance (5) low power consumption, etc.

ICL7109 can be easily connected to a variety of microprocessors without to add additional latches. ICL7109 uses a direct interface mode, when the ICL7109 conversion is over, the finish instruction is sent to the microcontroller by STATUS, single chip microcomputer read the data of the high byte and the low byte.

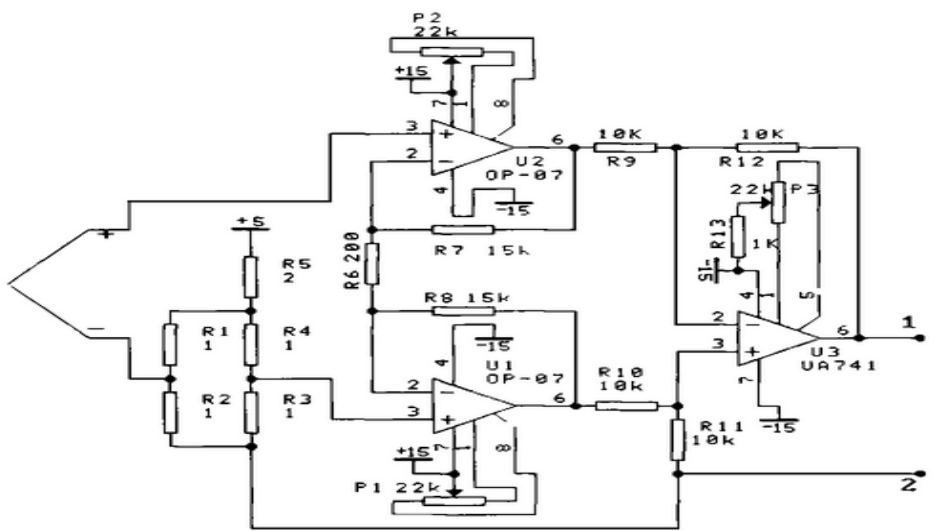

Fig. 3 hardware schematic diagram of temperature measurement system for thermocouple sensor

\section{Hardware design of trigger pulse circuit}

Because of the different voltage frequency in each region, we use the trigger pulse circuit to realize the voltage frequency control. As shown in the following figure, when the AC signal is zero 
(assuming this time is 0), a synchronized pulse is measured by the synchronous signal detection circuit. In a period, the P1.0 has remained high until the next sync pulse appears, causing disruption to start the next cycle, thus controlling the duty cycle of PWM.

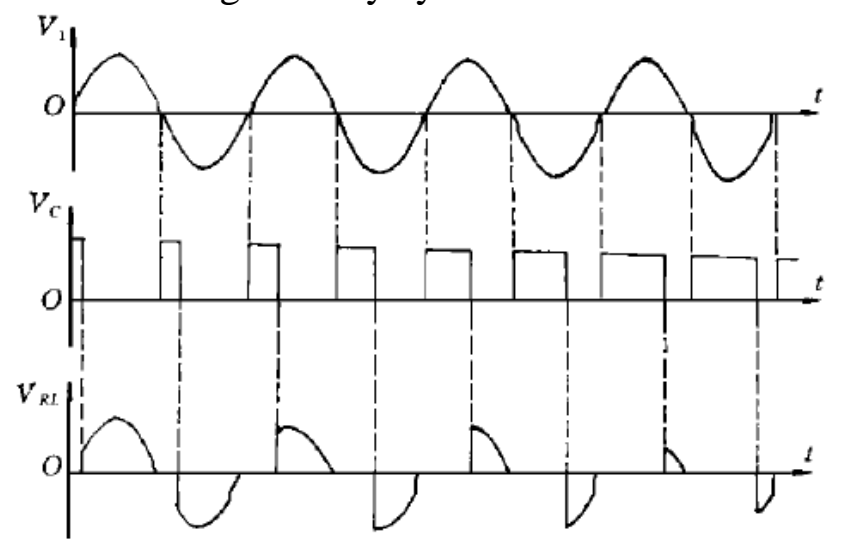

Fig.4 The different voltage frequency in each region

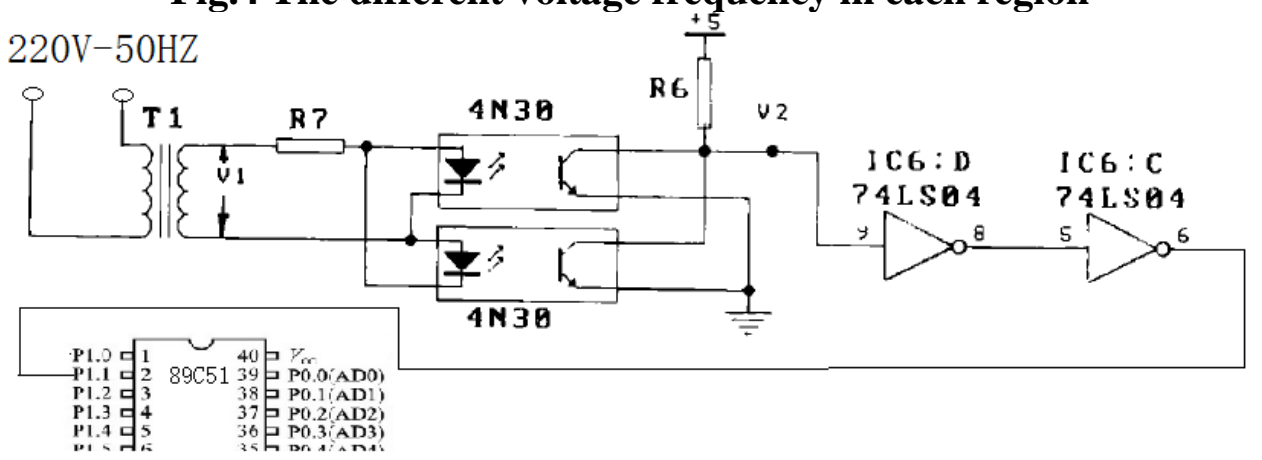

Fig.5 trigger pulse circuit diagram

\section{Hardware design of (heating) control circuit}

The control circuit is controlled by a single chip microcomputer, and the heating element is controlled by the pulse width modulation principle, This is because the pulse width modulation is the use of the digital output signal generated by the microprocessor to control the analog circuit. It is a very effective technology, widely used in many fields of measurement like communication, power control and signal conversion,etc.

Pulse width modulation (PWM) basic principle:

The control mode is controlled by the on-off of the switching device of the inverter circuit, then the output terminal is obtained by a series of equal amplitude pulses, using these pulses instead of a sine wave or the required waveform. That is, in the output waveform of the half cycle to generate multiple pulses, so that the equivalent voltage of each pulse is a sinusoidal waveform. According to certain rules, we can modulated the width of each pulse so that the output voltage of the inverter circuit and its output frequency can all be changed.

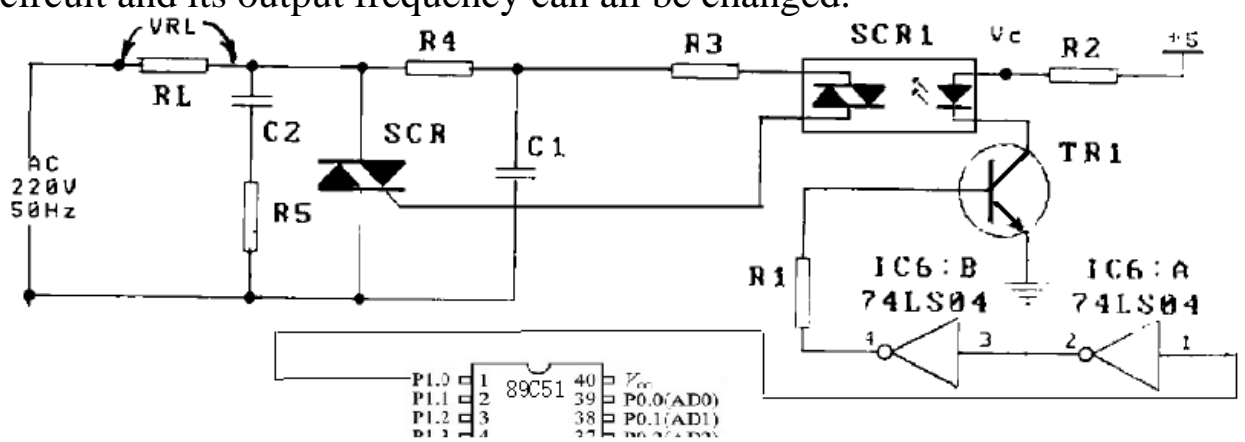

Figure 6 SCR control circuit

Since power is connected to the AC current of $20 \mathrm{~ms}$, the cycle is $220 \mathrm{~V} 50 \mathrm{HZ}$. By PWM modulation, the output is equal amplitude pulses, using a pulse clock cycle to control the AC output voltage, silicon controlled rectifier conduction angle and the voltage at the two ends of the electric 
heating wire size, so that we can control the heating speed of the electric heating wire. When the device starts, we need to make the electric heating wire heating rapidly, so we can using the MCU to control the PWM's output, so that the $220 \mathrm{~V}$ alternating current output full cycle. At this time, the two ends of the heating wire voltage reached the maximum value and maximum heating power, the temperature rise fastest, when the temperature is about to reach the set temperature, the PWM output clock period becomes shorter, at this point the AC output half cycle or even lower, two ends of the heating wire voltage decreased, both its power and heating speed reduced. When reaching the set temperature, the SCR control circuit disconnect, the heating wire stop heating, steam temperature drops, through temperature measurement circuit to detect steam temperature at this time, when less than set temperature 5 degrees or so, or single chip microcomputer timer reaches the setting time, control MCU output again, let the electric heating wire, repeat the above process, thermostatic steam therapy effect.
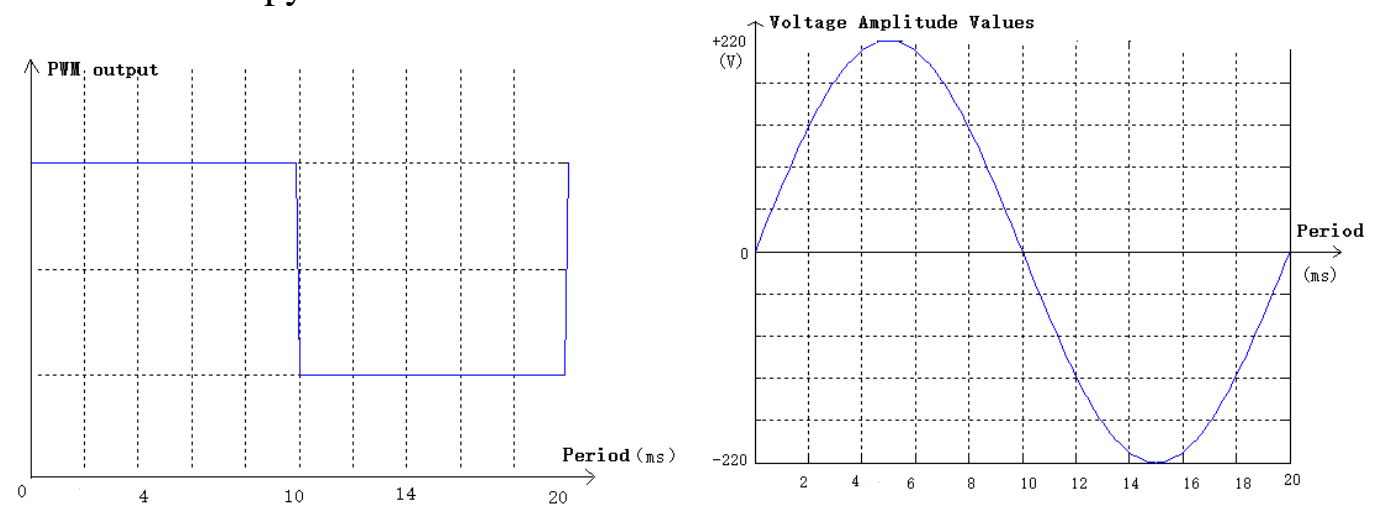

PWM output as shown

the power waveform diagram as shown

To control the PWM pulse output duty cycle by controlling the turn-on angle of silicon controlled rectifier, thus controlling the output range of the supply voltage and the size of the control voltage of the heating wire, which can control the heating rate and temperature.
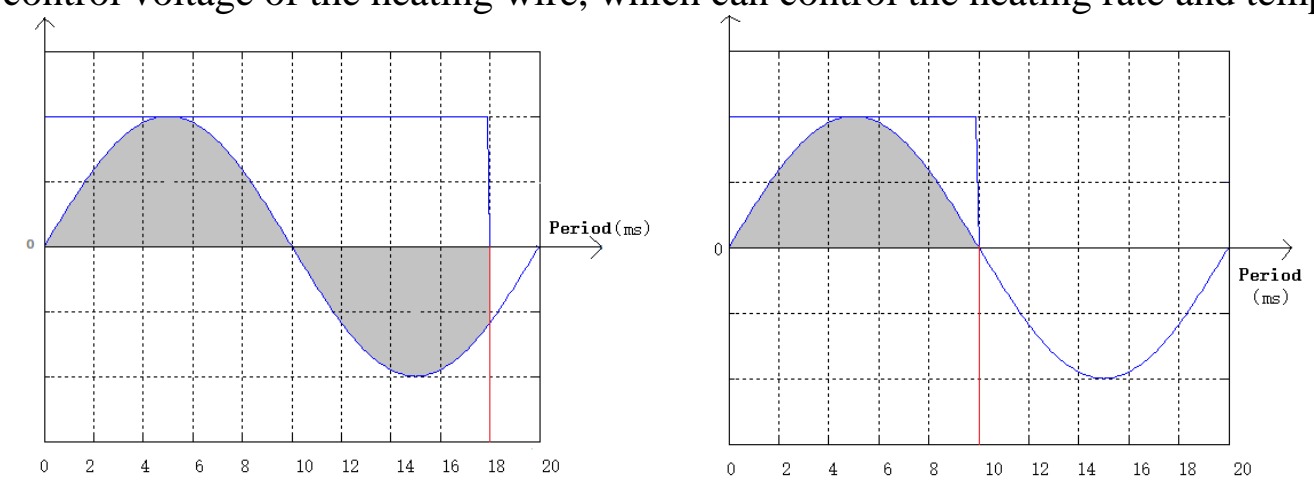

The voltage output which Output duty ratio is $80 \%$ Voltage output which Output duty ratio is $50 \%$

\section{The Software Design for The System}

Main program software design process:

When the device is enabled, we first processing the CPU initialization, the monitor initialization and self-inspection , after the interruption, the keyboard is kept in the scan state. 


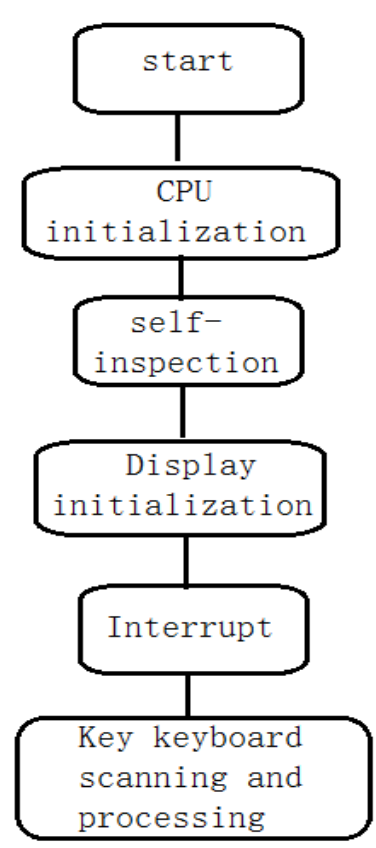

Main program software flow chart

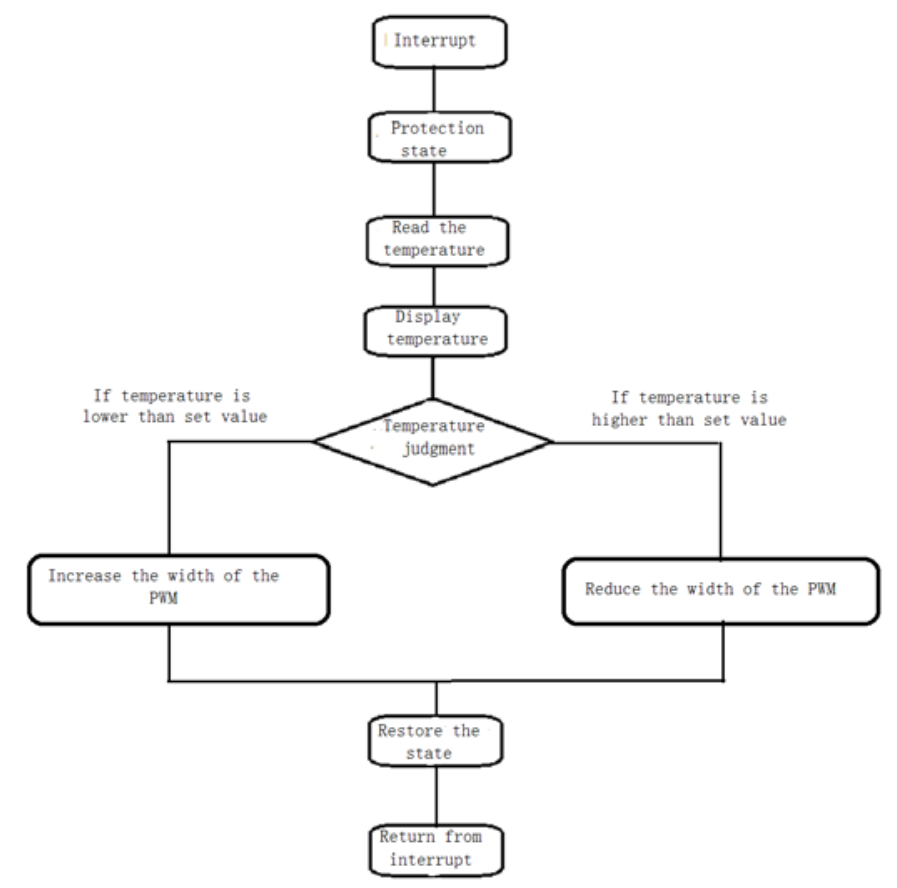

Interrupt service flow chart

Interrupt service program design process:

When entering the interrupt service routine, the system reads the temperature value in the break time, and compares with the setting temperature value, if the temperature is lower than the set temperature, the conduction angle of the silicon controlled rectifier can be increased, and the PWM width is expanded, On the other hand, reduce the PWM width, and then exits the interrupt, repeat the above process to achieve control of the temperature.

\section{Conclusion}

In order to meet the needs of the People's Daily health care, solve social "aging" problem faced by health problems, and improve the quality of people's lives, this subject has developed a kind of home care system which can be used in the scope of the community, This topic has done the main work is as follows:

(1) Through the analysis of the drawbacks of existing physical therapy instrument, this paper proposes a new type of family oriented clinical physiotherapy equipment. And the concrete scheme has carried on the detailed planning of the system.

(2) We use the steam temperature control system as the center of the Chinese medicine, traditional Chinese medicine fumigation device hardware platform was implemented.

The emergence of traditional Chinese medicine fumigation device, change the existing mode of clinical therapy. In this new medical mode, patients themselves become doctors, and they can complete the treatment at home. This patient-centered new medical model has the advantages of:

(1) Shorten the distance between doctor and patient, the patient oneself can become a doctor, and can be treated timely, reduce the delay of the illness.

(2) If the elderly and disabled people has this set of equipment, they can be treated independently at home, they also can achieve the result that should have, and avoids the journey to the hospital.

(3) Even the health of workers also can undertake this health care treatment, through the device can make the disease in the bud, and have health care function,

(4) This device can be widely used in hospitals of physiotherapy, the pain, regions, orthopedics, dermatology, gynecology and internal medicine, etc., at the same time can also be applied to nursing homes, rehabilitation centers, community health care and beauty weight watchers, and so on. 


\section{References}

[1] Jing li, Xin gu. "physical therapy instrument application in geriatrics and prospects". Health care equipment, 2000, 20(3): 28-30

[2] Yu-Dong Shuai. Medical diagnosis of electronic instruments and technology [M]. Electronic industry press.

[3] De-zhu Mao. New semiconductor devices and its applications [M]. Electronic industry press, 2002, 12:267

[4] Wei-zheng Jin. The principle and application of the single line digital temperature sensor [M]. Electronic technology applications, 2000, (6) : 66-68.

[5] Yi-hong Li , Ai-qin Wang. Chinese medicine fumigation treatment of neck and shoulder bed lumbocrural pain [M]. Qingdao pharmaceutical and health care, 2004 (3) : 33.

[6] Ming-yan wang. Chinese medicine fumigation bed treatment nursing experience in joint disorders [M]. The modern nursing journal. (7) : 2006-57 to 56

[7] Yi-hong Li , Ai-qin Wang.Chinese medicine fumigation bed used in the treatment of neck and shoulder lumbocrural pain patient care [J]. Zhonghua, 2004, (10) : 1510

[8] Wei-zhuang jiang.Ridge source sex lumbocrural pain. The first edition. Beijing: Beijing people's medical publishing house, 2002:38.

[9] Shi yan.Digital electronic technology foundation [M]. 4. Beijing: higher education press, 2002.

[10] Chao-qing li.Single chip microcomputer principle and interface technology [M]. 3 edition. Beijing: Beijing university of aeronautics and astronautics press, 2005.

[11] Hui cao.The design of digital temperature measurement circuit and its realization of microelectronics, 2001, 31 (3) : 229-232. 